\title{
Research Paper \\ Prevalence and Gender Differences of Elder Abuse among Community-dwelling Older Adults in Tabriz, Iran
}

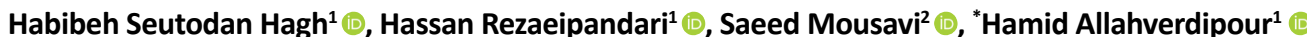

1. Department of Health Education \& Promotion, Faculty of Health, Tabriz University of Medical Sciences, Tabriz, Iran.

2. Department of Statistics and Epidemiology, Faculty of Health, Tabriz University of Medical Sciences, Tabriz, Iran.

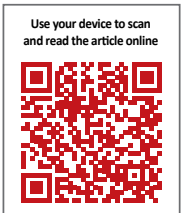

Citration: Sutodan Hagh H, Rezaeipandari H, Mousavi S, Allahverdipour H. [Prevalence and Gender Differences of Elder Abuse among Community-dwelling Older Adults in Tabriz, Iran (Persian)]. Iranian Journal of Ageing. 2021; 15(4):458-471. https://doi.org/10.32598/sija.15.4.1124.1

Keywords:

Older adults, Elder abuse, Gender difference, Neglect, Prevalence

\section{A B STRACT}

Objectives Elder abuse is a phenomenon that has become more apparent with the increase in aging population, but there are insufficient evidences about the prevalence of elder abuse and gender differences in this area. Therefore, this study aims to investigate the prevalence of elder abuse and its gender differences among community-dwelling older adults in Tabriz, Iran.

Methods \& Materials In this cross-sectional study, 414 older adults aged $\geq 60$ years were recruited from health complexes and centers in Tabriz, Iran in 2018 by using random sampling method. Data collection tools were a demographic form and the Domestic Elder Abuse Questionnaire which were completed through interview. The collected data were analyzed in SPSS V. 22 software.

Results It was reported that $52.6 \%$ had experienced abuse by family members. Emotional neglect was the most common type of elder abuse (26.6\%) followed by financial abuse (17.6\%). Physical abuse was the least common type of elder abuse. Except for financial abuse, older women had experienced more abuse than older men. Of those who were abused, only $31.7 \%$ recognized it as elder abuse.

Conclusion Most of older adults experience emotional neglect and financial abuse from family members and perceive them as their normal behaviors. Older women experience more abuse than older men. It is necessary to prevent elder abuse and familiarize older adults with different types of elder abuse.

\section{Extended Abstract}

\section{Introduction}

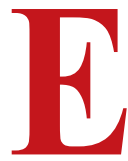

lder abuse is a phenomenon that has become more apparent with the rapid increase in the number of elderly [1]. According to statistics provided by the World Health Organization, the prevalence of this phenomenon in developed countries varies from 1 to $10 \%$ [2]. Gender is an important factor in dealing with abuse. About $10 \%-15 \%$ of older women worldwide are exposed to physical abuse $[3,4]$. Considering the different dimensions of elder abuse, it can be stated that many elderly people have been abused by their caregivers and those around them at least once [5]. However, there is insufficient evidence on the prevalence of elder abuse and its gender pattern among the elderly population in Iran. Therefore, this study aims to investigate the prevalence of elder abuse and related patterns based on gender differences in the elderly population in Iran

\section{Methods \& Materials}

This descriptive-analytical study was conducted on 414 elderly people aged $\geq 60$ years in Tabriz, Iran who were recruited from health complexes and centers. Inclusion cri-

\section{* Corresponding Author:}

Hamid Allahverdipour, PhD.

Address: Department of Health Education \& Promotion, Faculty of Health, Tabriz University of Medical Sciences, Tabriz, Iran.

Tel: +98 (914) 3147363

E-mail: allahverdipour@gmail.com 
teria were age $\geq 60$ years, resident of Tabriz city, living in their own home or at the home of a close relative, living with at least one family member, willingness to participate in study and answering questions, and no any cognitive or auditory impairment (full awareness of time, place and person). Two-stage cluster sampling method was used to select samples, where health complexes were as first cluster and health centers as secondary cluster. After random selection of first cluster, two centers from each cluster were randomly selected and in proportion to the population of the health complex, the samples from the health center were randomly selected. After coordination with the Health Organization of Tabriz city and obtaining the necessary permits, were referred to the study centers and the elderly were randomly selected using the SIB system (http://sib.sbmu.ac.ir). After explaining the research process on the phone, if the selected elderly had the conditions to enter the study, s/he was invited to participate in the study; if not, another elderly from the same center was replaced. Data collection tools was a demographic form, and the Domestic Elder Abuse Questionnaire which has 49 items and 8 subscales. They were completed through interview and the collected data were then analyzed in SPSS V. 22 software.

\section{Results}

A total of 414 elderly people (261 females and 153 males) aged 60-98 years (mean age $=67 \pm 7.09)$ participated in this study. The majority of participants were married (73\%) and had their own houses $(92 \%) .52 .6 \%$ of participants reported abuse. The most common type of abuse was emotional neglect by family members; i.e. less visiting and calling them $(26.8 \%)$ or ignoring and not talking to them $(26.6 \%)$. Financial abuse $(17.6 \%)$ was in the second place. Physical abuse was the least common abuse. The results showed that, except for the financial abuse, older women were more abused than older men (Table 1). Participants complained that family members do not return the money they borrowed from them. In some cases, yelling at the elderly (15.5\%) and blaming them for no reason (14.5\%) by family members were reported as psychological abuse.

Table 1. Mean and standard deviation of different types of elder abuse categorized by gender

\begin{tabular}{|c|c|c|c|c|}
\hline Type of Elder Abuse & Gender & Mean \pm SD & Sig. & $\mathbf{F}$ \\
\hline \multirow{3}{*}{ Physical abuse } & Men & $4 \pm 0.65$ & \multirow{3}{*}{0.000} & \multirow{3}{*}{12.94} \\
\hline & & & & \\
\hline & Women & $10.39 \pm 2.20$ & & \\
\hline \multirow{3}{*}{ Financial abuse } & Men & $16.62 \pm 8.38$ & \multirow{3}{*}{0.042} & \multirow{3}{*}{4.17} \\
\hline & & & & \\
\hline & Women & $13.30 \pm 6.79$ & & \\
\hline \multirow{3}{*}{ Social rejection } & Men & $3.51 \pm 0.5$ & \multirow{3}{*}{0.24} & \multirow{3}{*}{5.11} \\
\hline & & & & \\
\hline & Women & $11.36 \pm 1.56$ & & \\
\hline \multirow{3}{*}{ Emotional neglect } & Men & $38.91 \pm 22.03$ & \multirow{3}{*}{0.057} & \multirow{3}{*}{3.64} \\
\hline & & & & \\
\hline & Women & $40.28 \pm 29.88$ & & \\
\hline \multirow{3}{*}{ Caring neglect } & Men & $13.53 \pm 3.51$ & \multirow{3}{*}{0.027} & \multirow{3}{*}{4.92} \\
\hline & & & & \\
\hline & Women & $15.12 \pm 5.9$ & & \\
\hline \multirow{3}{*}{ Financial neglect } & Men & $11 \pm 1.98$ & \multirow{3}{*}{0.001} & \multirow{3}{*}{23.88} \\
\hline & & & & \\
\hline & Women & $19.36 \pm 6.20$ & & \\
\hline \multirow{3}{*}{ Deprivation of parental authority } & Men & $5.66 \pm 2.42$ & \multirow{3}{*}{0.001} & \multirow{3}{*}{29.59} \\
\hline & & & & \\
\hline & Women & $8.69 \pm 4.61$ & & \\
\hline \multirow{3}{*}{ Psychological abuse } & Men & $17.34 \pm 8.14$ & \multirow{3}{*}{0.351} & \multirow{3}{*}{0.871} \\
\hline & & & & \\
\hline & Women & $19.07 \pm 9.02$ & & \\
\hline
\end{tabular}


Another important result of this study was the level of understanding of elder abuse and how to deal with it. For this purpose, three new questions were added to the end of the Domestic Elder Abuse Questionnaire, and those who were abused even with a positive answer were asked to answer "yes" or "no" to these questions. Out of 218 elderly people who were abused, only $69(31.7 \%)$ considered the mentioned abuses as elder abuse; 131 (60.1\%) of the abused elderly informed others about their problems. A high percentage $(91.7 \%)$ of the elderly were satisfied with their family members despite being abused.

\section{Conclusion}

Despite the importance of treating elders with respect, the elderly in Tabriz experience elder abuse in the family, which can be due to rapidly changing values, attitudes, behavioral patterns and moral standards in the family. They mostly experience financial abuse and neglect and perceived many instances of elder abuse to be normal behavior of family members. Furthermore, older women are more likely to be abused than males. It is necessary to take the necessary measures to inform families about the physical, psychological, emotional and social needs of the elderly, change negative attitudes towards the aging period, and attracting the attention of the country's health care system to increase respect for the elderly.

\section{Ethical Considerations}

Compliance with ethical guidelines

This study was approved by Ethics Committee of Tabriz University of Medical Sciences (Code: IR.TBZMED. REC.1398.191). All ethical principles are considered in this article. The participants were informed about the purpose of the research and its implementation stages. They were also assured about the confidentiality of their information and were free to leave the study whenever they wished, and if desired, the research results would be available to them.

\section{Funding}

This study was extracted from the Msc. thesis of first author at Department of Health Education \& Promotion, Faculty of Health, Tabriz University of Medical Sciences, Tabriz. Also, this study was supported by Saba Pension Strategies Institute.

\section{Authors' contributions}

Conceptualization: Habibeh Seutodan Hagh, Hassan Rezaeipandari, Hamid Allahverdipour; Methodology:
Habibeh Seutodan Hagh, Hassan Rezaeipandari, Hamid Allahverdipour, Saeed Mousavi; Data analysis: Hassan Rezaeipandari, Hamid Allahverdipour, Saeed Mousavi; Writing of original draft and editing: Habibeh Seutodan Hagh, Hassan Rezaeipandari, Hamid Allahverdipour; Final approval: All authors.

\section{Conflicts of interest}

The authors declare no conflict of interest.

\section{Acknowledgements}

The authors would like to thank Saba Pension Strategic Institute (SABA) for financial support. The authors also appreciate the assistance of the Health Center of Tabriz City. 


\title{
فراوانى سالمندآزارى و التَوى جنسيتى آن در جمعيت سالمند ساكن در مناطق شهرى تبريز
}

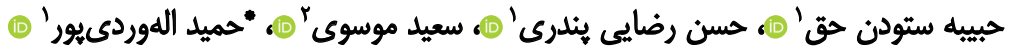 \\ ا. كروه آهوزش بهداشت و ارتقاى سلامت، دانشكده بهداشت، مانشعاه علوم يزشكى تبريز، تبريز، ايران.

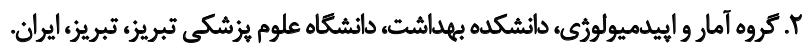

\begin{abstract}
حكبن

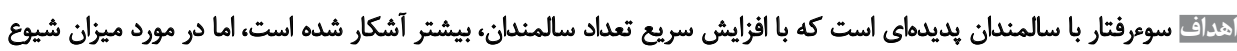

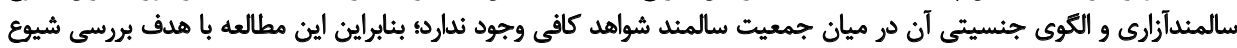

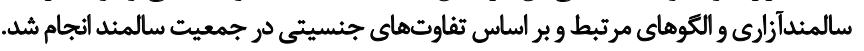

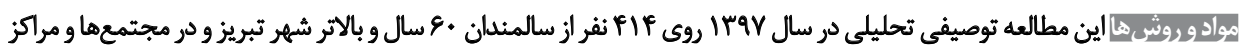

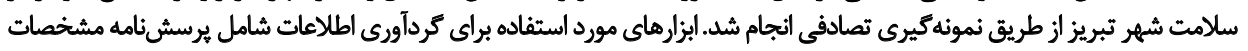

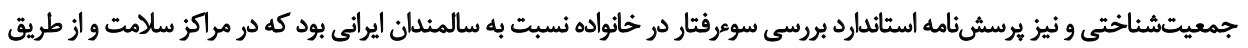

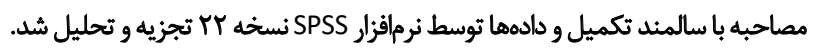

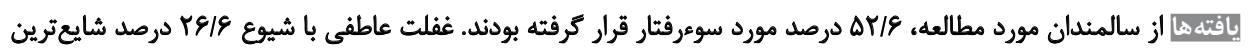

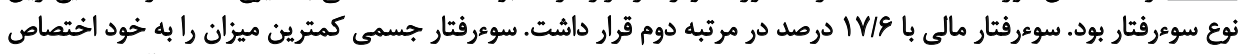

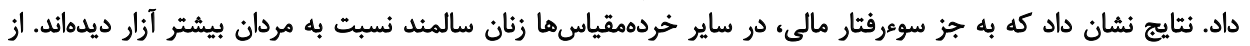

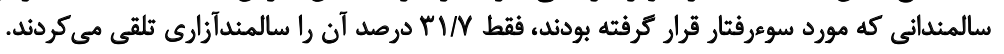

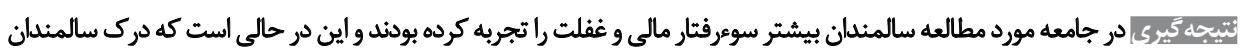

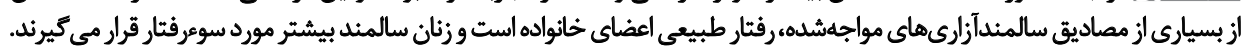

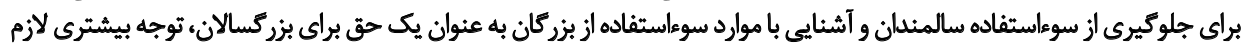

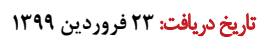

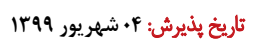

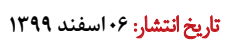

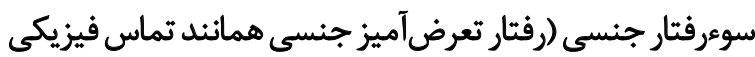
مقدمه

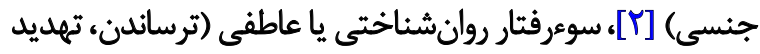

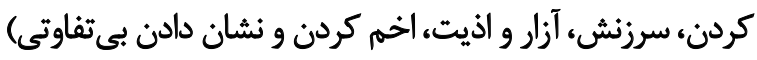

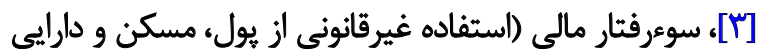

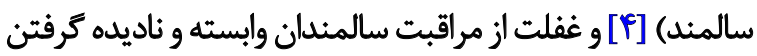

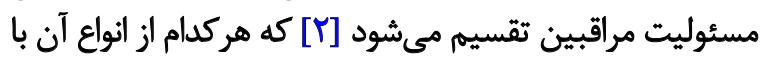
علاثم مربوطه قابل شناسايى است.

هروى و همكاران طى انجام مطالعهاى به تبيين تئوريك

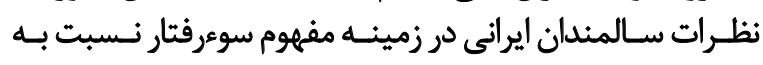

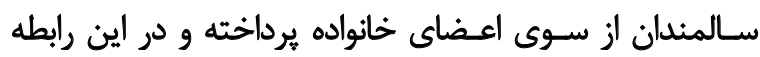

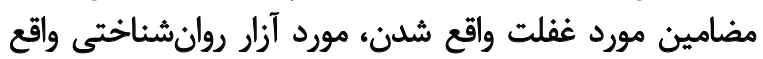

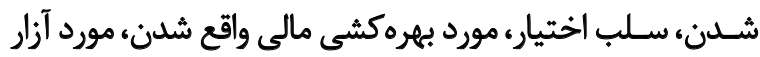
بدنى واقع شدن و طرد شدن را استخراج كردند [هـ]

بيشتر از دو دهه است كه سوءرفتار با سالمندان مانند ديكر

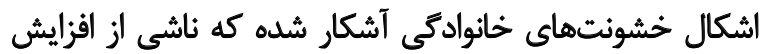

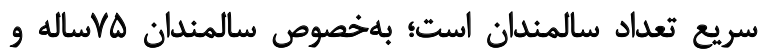

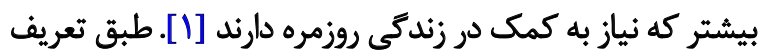

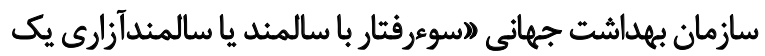

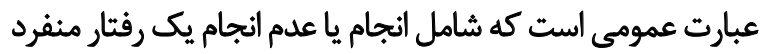

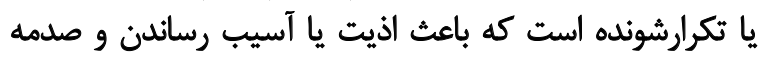

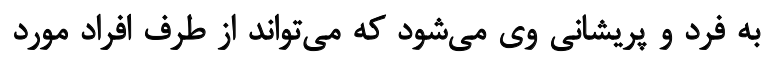

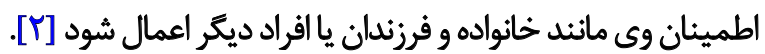

بر اساس جديدترين طبقهبندى، سالمندآزارى از نظر محققان

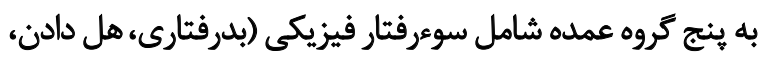

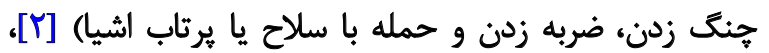

* نويسيده مسئول: دكتر حميد الدوردىيوري

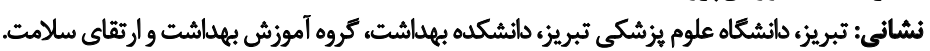

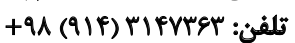
allahverdipour@gmail.com بلعن الكترونيكي: 


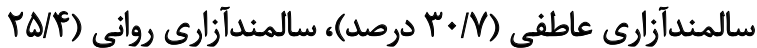

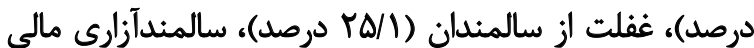

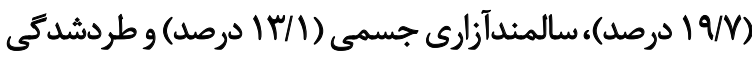

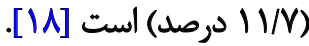

همجنين كزارش شده است كه اعضاى خانواده بيشترين موارد

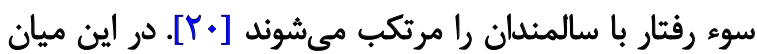

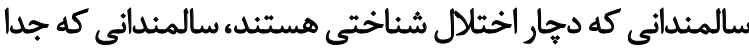

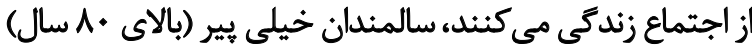

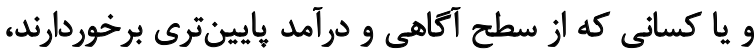

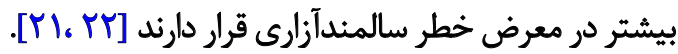
نتايج اين مطالعات نشان مي دهد على غئر حضم حضور نسبتاً بالايى

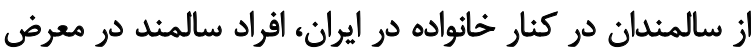

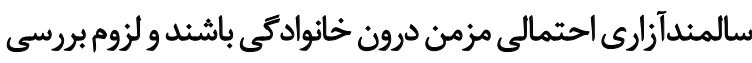

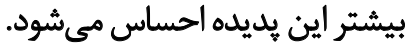

عليرغم تأكيد سازمان بهداشت جهانى مبنى بر لزوم تشخيص و

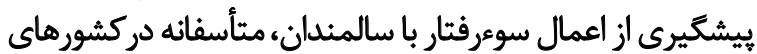

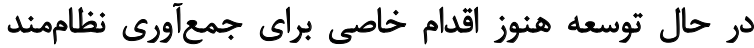

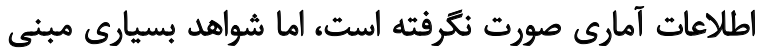

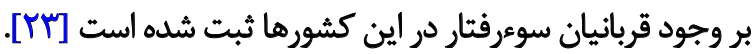
در ايران با توجه به وجود مصوبه هيئت وزيران مبنى بر اتخاذ

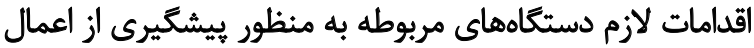

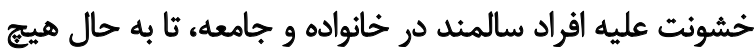

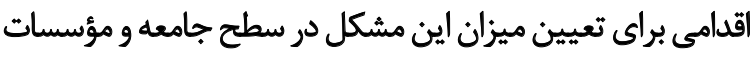

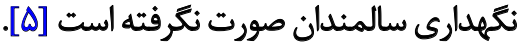
نظر به اينكه در مورد ميزان شيوع سالمندآزارى و الكوى

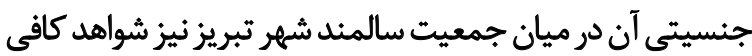

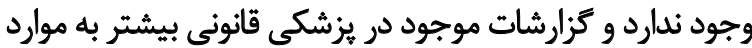

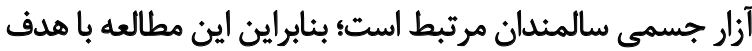

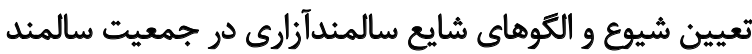

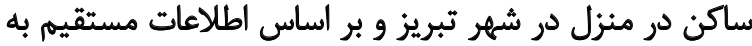

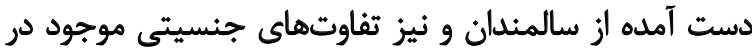
الكوهاى سالمندآزارى انجام شد.

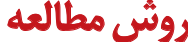

اين مطالعه توصيفى تحليلى از نوع مقطعى بود كه در زمستان

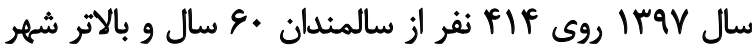

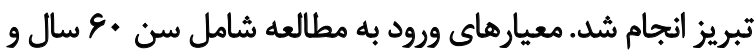

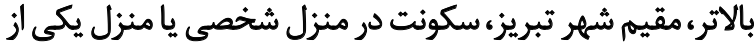

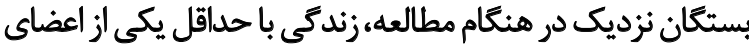

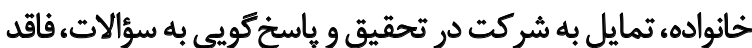

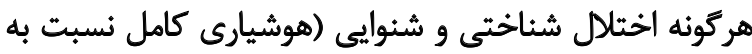

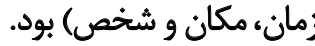

همجينين در سالمندانى كه مورد سوءرفتار قرار مي كيرند احتمال

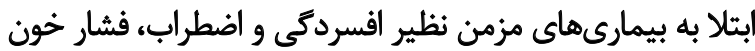

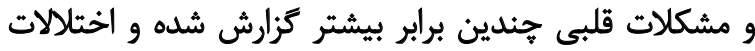

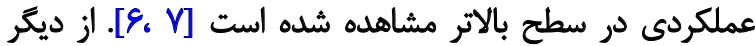

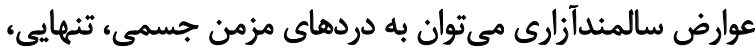

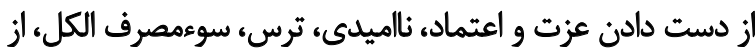

دست دادن اموال، آسيب جسمانى و معلوليت اشاره كرد [1]]. بر اساس مطالعات انجام كرفته در زمينه سالمندآزارى، ميزان

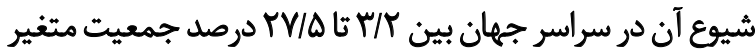

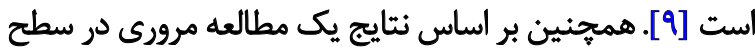

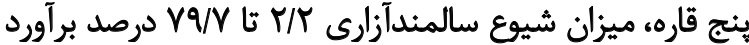

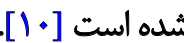

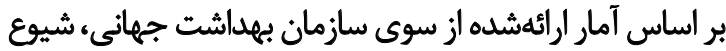

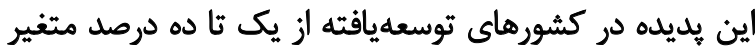

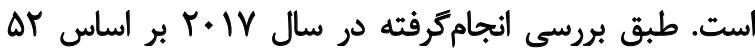

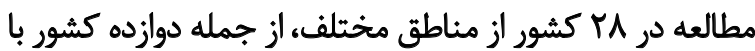

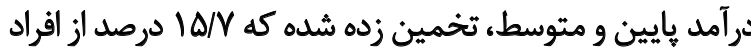

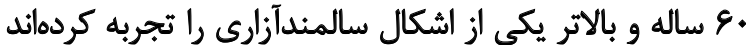

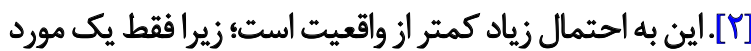

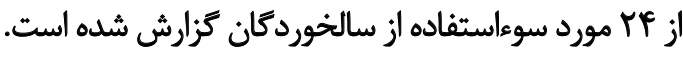
عوامل مختلفى بر اين يديده اثركذار است كه يكى از آنها

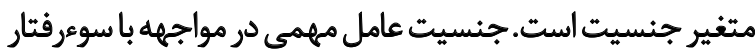

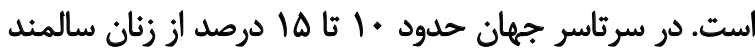

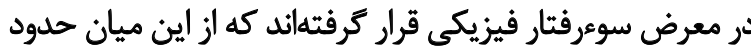
If

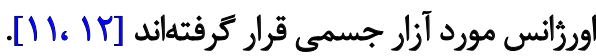
مطالعهاى كه در سال | 9 | أدر بيمارستانى در شهرستان ميبد

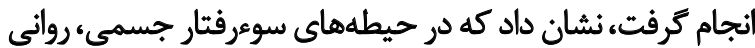

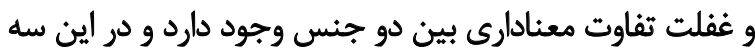

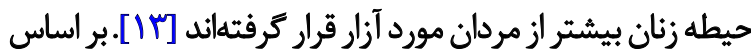

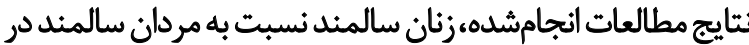

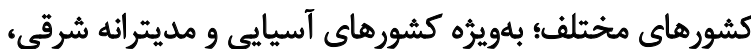
بيشتر مورد سوءرفتار قرار مي كيرند [1f

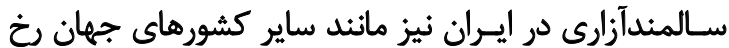

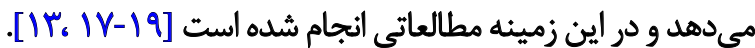

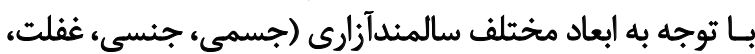

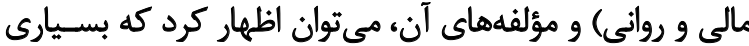

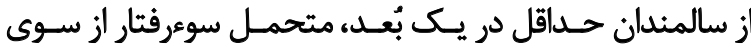

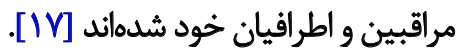
يرؤششى كه توسط مولاييى و همكاران انجام گرفته، نشان داد

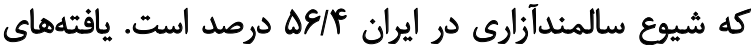
اين يُروهش نشان داد كه شايعترين نوع سالمندآزارى به درات ترتيب يافتهاي 
كويههاى ابزار مذكور داراى كزينههاى "(بلى" و "اخيره) و

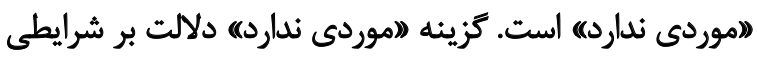

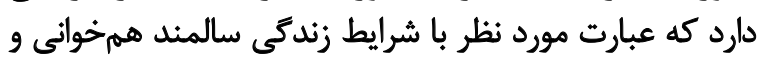

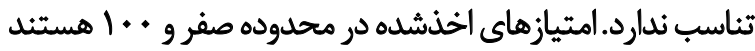

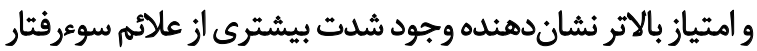

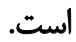

ويروّى هاى روانسنجى ابزار مذكور بررسى شده است و از

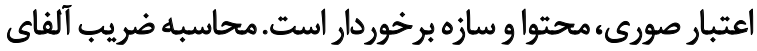

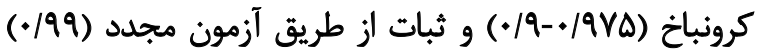

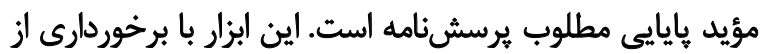

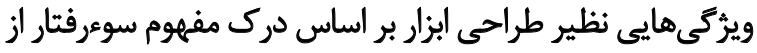

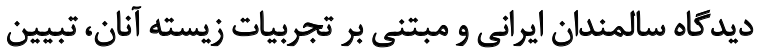

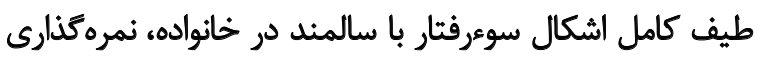

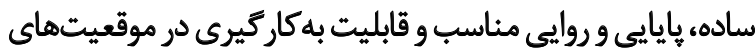

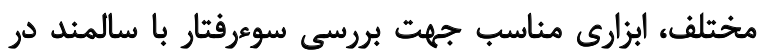

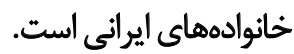

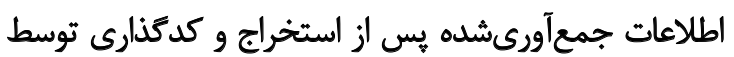

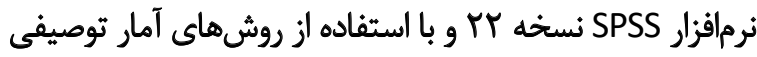

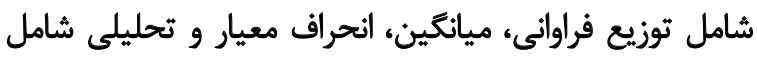
آزمون تى مستقل و آناليز واريانس تجزين انجراف معيار وتحليل شد. تحليل

يافتهاه

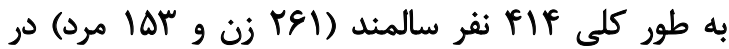

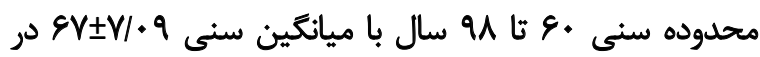

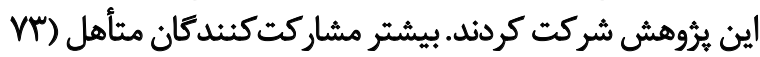

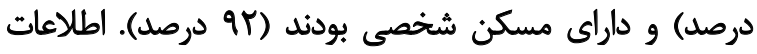
جمعيتشناختى شركت كنندكان در جدول شماره

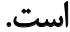

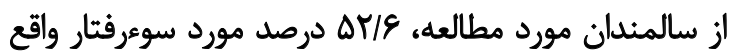

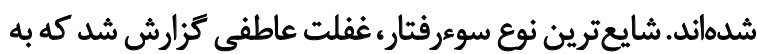

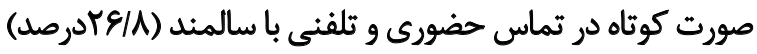

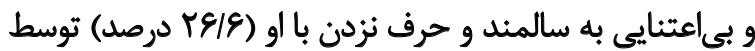

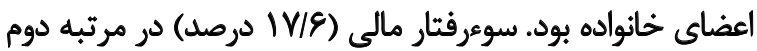

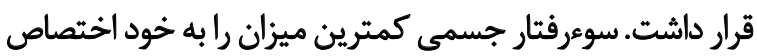

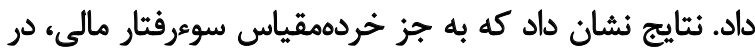

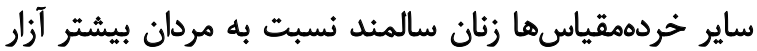

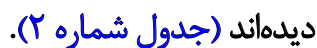

سالمندان از عدم بازستاندن قرض كرفتنهشده توسط اعضاى

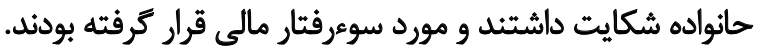

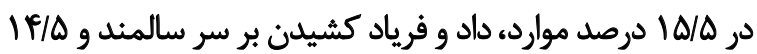

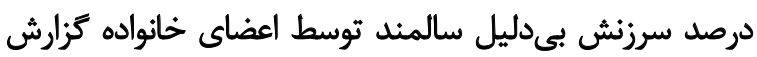

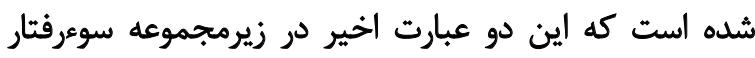

سالمندانى كه قادر به هاسخ كويى به سؤالات نبودند و سالمندانى

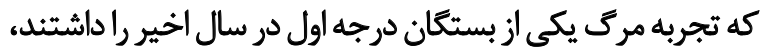

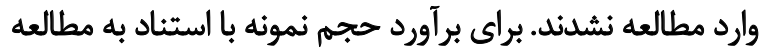

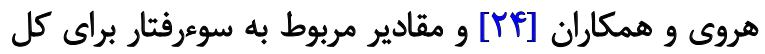

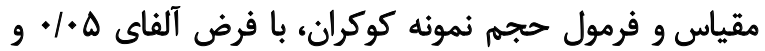

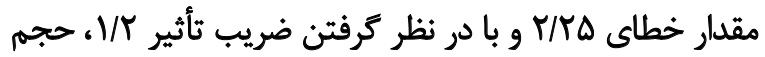

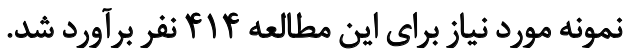

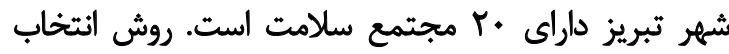

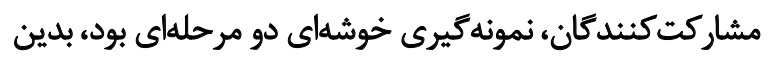

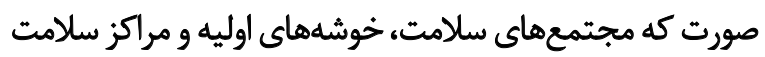

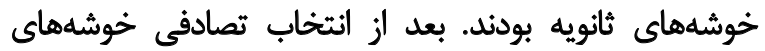

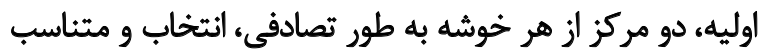

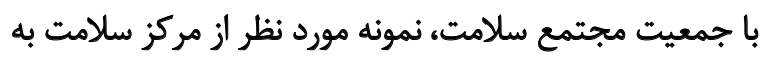
صورت تصادفى ساده انتخاب شد.

يس از هماهنكى با مركز بهداشت شهرستان تبريز و اخذ ماخد

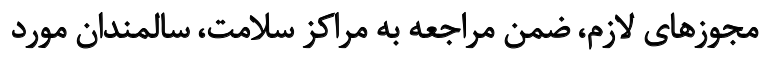

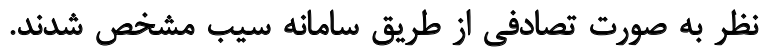

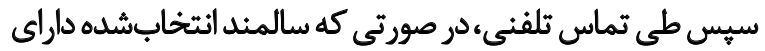

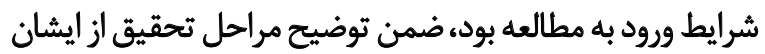

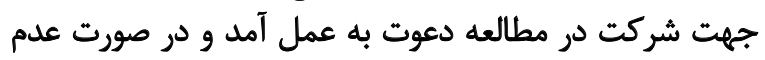

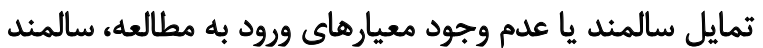
ديكرى از همان مركز جايكزين شدان

براى تكميل يرسشنامهها در صورت باسواد بودن سالمند،

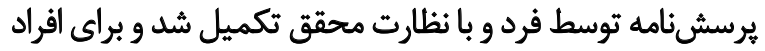

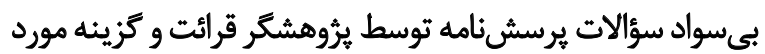

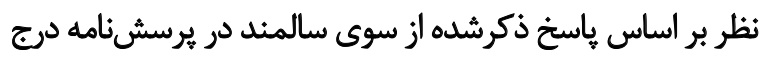

كردآورى اطلاعات در اين مطالعه توسط دو ابزار انجام شد:

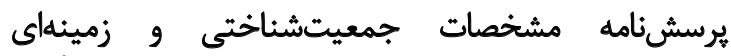

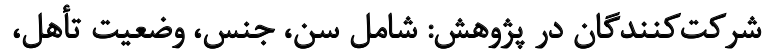

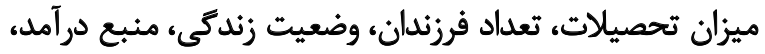

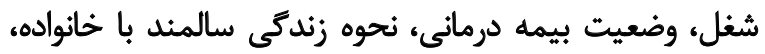

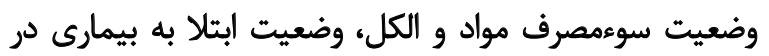
كذشته و حال و وضعيت خواب سالمند بود. يرسش نامه استاندارد سوءرفتار در خانواده نسبت به سالمندان

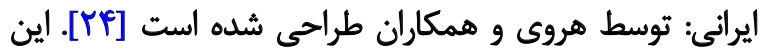

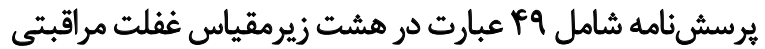

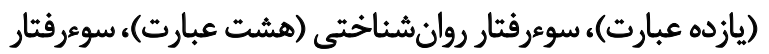

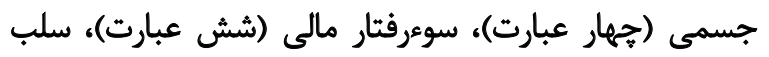

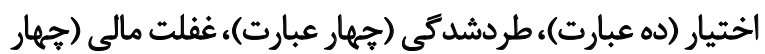
عبارت) و عاطفى (دو عبارت) است. 
جدول ا. توزيع فراوانى متغيرهاى جمعيتشناختى و زمينهاى در سالمندان مورد بررسى

\begin{tabular}{|c|c|c|c|}
\hline \multicolumn{2}{|c|}{ تعداد (درصد) } & \multirow{2}{*}{ حالت } & \multirow{2}{*}{ متغير } \\
\hline خير & بله & & \\
\hline$V \wedge(r q / \Lambda)$ & $V \Delta(m e / P)$ & مرد & \multirow[b]{2}{*}{ 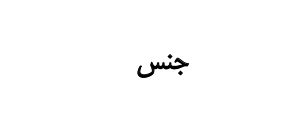 } \\
\hline $114(\varepsilon \cdot \pi)$ & $\operatorname{lif}(8 \Delta / 8)$ & ij & \\
\hline$\Delta \Delta(T A)$ & $\Delta V(T \& / T)$ & - ل مجرد & \multirow{2}{*}{ وضعيت تأهل } \\
\hline IfI $(N)$ & $|8|(N / A)$ & مثتأهل & \\
\hline$P \Delta(M T)$ & $\operatorname{Pr}(Y / \Delta)$ & بيىسواد & \multirow{5}{*}{ ميزان تحصيلات } \\
\hline$\Delta q(r \cdot 11)$ & $V \cdot(m / N)$ & إبتداييى & \\
\hline$r \cdot(1 \cdot \pi)$ & $M(I F / T)$ & راهنمائي & \\
\hline $\operatorname{rr}(M / R)$ & $M e(1 \Delta / 8)$ & متوبسطاه و دييلم & \\
\hline$r \cdot(1 \Delta / T)$ & $r q(\mid q / 9)$ & دانشكاهى & \\
\hline$r \cdot(\Lambda \Delta / \Psi)$ & $\operatorname{IV}(V / A)$ & شاغل & \multirow{4}{*}{ وضعيت اشتغال } \\
\hline $91(\$ \& / 4)$ & $\operatorname{IrT}(\Delta \&)$ & خانهدار & \\
\hline$g q(r \Delta / T)$ & $\varepsilon_{\lambda}(\mu / / T)$ & بازنشسته & \\
\hline$\varepsilon(\% / l)$ & $11(\Delta)$ & بيكار & \\
\hline (1) $(9 T / \pi)$ & $r+r(9 T / V)$ & شخصى & \multirow{3}{*}{ وضعيت تملك مسكن } \\
\hline$I f(V / T)$ & $V(\Gamma / T)$ & استيجارى & \\
\hline $1(* / \Delta)$ & $9(\% / 1)$ & مسكن فرزندان & \\
\hline$\Delta q(\varphi \Delta / \varphi)$ & $A \in(r q / \Delta)$ & با همسر & \multirow{6}{*}{ وضعيت زندكى سالمند با خانواده } \\
\hline$P V(M P)$ & EN $(r / T)$ & همسر و فرزند & \\
\hline $10(V / F)$ & $18(N / T)$ & فرزند يسر & \\
\hline $9(\varphi / 8)$ & $I r(\Delta / \Delta)$ & فرزند دختر & \\
\hline$\Delta(r / \Delta)$ & $1(\cdot / 4)$ & اقوام درجه يك & \\
\hline$M(1 \Delta / q)$ & $\mathrm{ra}(\mid g / 1)$ & مرخشى & \\
\hline $1 T \Delta$ (\&N9) & $\operatorname{lQV}(N)$ & حقوق بازئشستكى & \multirow{5}{*}{ مجل درآهد سالمند } \\
\hline $\operatorname{le}(\pi / \Delta)$ & $r(I V)$ & شعل آزاد & \\
\hline$M(\& / 1)$ & $19(N)$ & كمى فرزندان & \\
\hline$\cdot(\cdot)$ & $r(\cdot / 9)$ & كمى اقوام & \\
\hline$r(V / \Delta)$ & $r(1 / /)$ & هؤسسات حمايتى & \\
\hline $\operatorname{IrT}(\mathrm{AV} / \mathrm{A})$ & $199(91 / 7)$ & دارد & \multirow[b]{2}{*}{ وضعيت بيمه } \\
\hline$M E(I T / T)$ & $19(\mathrm{NV})$ & 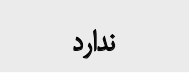 & \\
\hline$N \Delta(f T / T)$ & $\| V(\Delta r / V)$ & 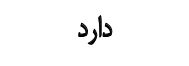 & \multirow{2}{*}{ سابقه ابتلا بله بيمارى در كذشته } \\
\hline 111 (rg/g) & $1.1(f \& / \pi)$ & ندارد & \\
\hline $1.9(\Delta \Delta / 9)$ & $\log (V / \Delta)$ & دارد & \multirow{2}{*}{ ابتلا به ييمارى كنونى } \\
\hline AV $(f F / F)$ & ET (TND) & ندارد & \\
\hline
\end{tabular}


جدول r. توزيع ميانكين و انحراف معيار انواع سوءرفتار بر حسب جنس در سالمندان مورد بررسى

\begin{tabular}{|c|c|c|c|c|}
\hline $\mathbf{F}$ & $\mathbf{P}$ & ميائيين土انحر اف معيار & جنسيت & نوع سوءرقتّار \\
\hline $\mid r / 9 F$ & $\%$ & $\begin{array}{c}r \pm+/ 80 \\
1 . / R q \pm r / r\end{array}$ & زرد & سوءرفتار جسمى \\
\hline$F / / Y$ & $.1 \cdot H T$ & $\begin{array}{l}\mid G / G Y \pm N H A \\
\mid r / r \cdot \pm \& / V q\end{array}$ & زن & سوعرفتار \\
\hline$\Delta / 11$ &.$/ M$ & $\begin{array}{l}r / \Delta \mid \pm \cdot / \Delta \\
11 / R r \pm 1 / \Delta s\end{array}$ & هرد & طردشدىى \\
\hline m/at & $.1 \cdot \Delta V$ & $\begin{array}{l}r N q 1 \pm r r / \cdot r \\
r \cdot / r A \pm r q / M\end{array}$ & زرد & غُفلت عاطفى \\
\hline P/ar & $.1 \cdot \mathrm{rV}$ & $\begin{array}{l}|r / \Delta r \pm r / \Delta| \\
|\Delta /| r \pm \Delta / q\end{array}$ & مرد & غفلت مراقبتى \\
\hline$r / M$ & $>\cdot 1 \cdot+1$ & $\begin{array}{l}11 \pm 1 / 41 \\
12 / r s \pm s / r\end{array}$ & زرد & غفلت مالى \\
\hline rq/aq & $>\cdot 1 \cdot+1$ & $\begin{array}{l}\Delta / \& \& \pm T / R T \\
N \& q \pm F / \& I\end{array}$ & مرد & سلب اختيار \\
\hline - $\mid A n$ &.$|r \Delta|$ & $\begin{array}{l}|V / M E \pm N| F \\
\mid V / V \pm V \cdot r\end{array}$ & 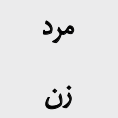 & سوءرفتار روان \\
\hline
\end{tabular}

L

خانكى است كه بيشينهاي بسيار قديمى دارد، اما در دو دها اخير

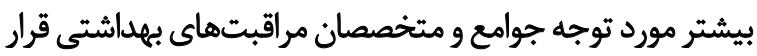

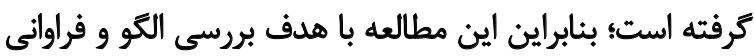
سالمندآزارى در جمعيت سالمند ساكن در منزل درائ در شهر تبريز

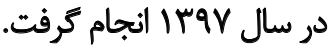

يافتههاى حاصل از اين يروهش نشان داد كه بيش از نيمى از

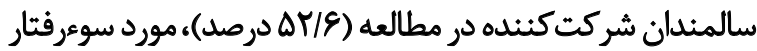

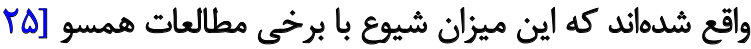

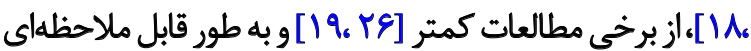

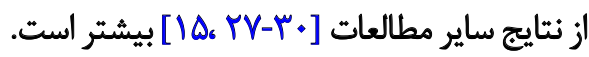

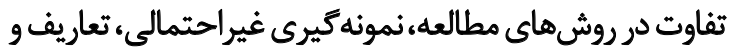

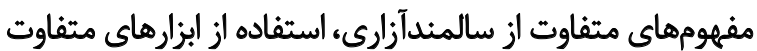

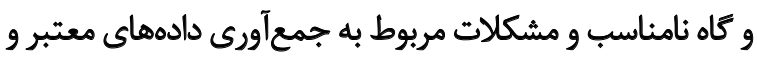

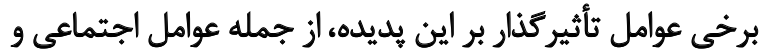

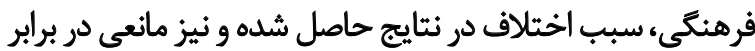
مقايسه علمى نتايج يديد آمده است. اما آنجه به روشنى مشهود است، آن است كه درصدي سالمندآزارى در مطالعات انجامشده در ايران و تبريز نسبت به آنه دردي آنس
روانشناختى قرار گرفتهاند (جدول شماره ب). يكى ديكر از نتايج مهمم اين مطالعه، ميزان درك سالمندان

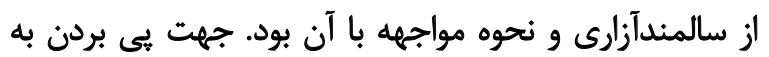

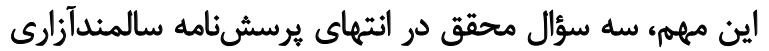

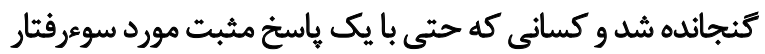

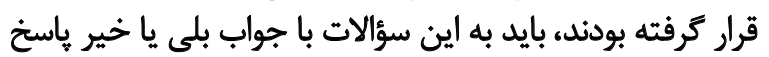

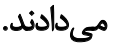

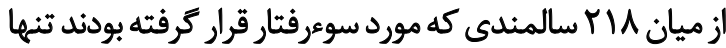

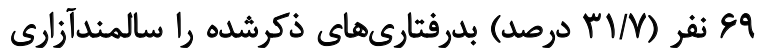

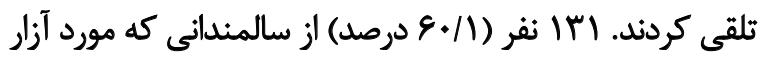

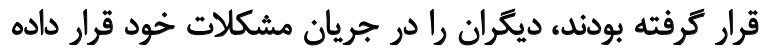

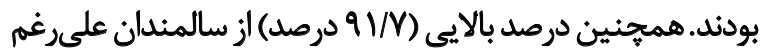

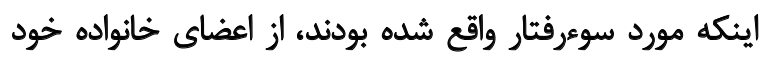
رضايت داشتند (جدول شماره F).

$$
\text { بـ }
$$

سالمندآزارى كه به عنوان يك تهديد جدى در افراد بالاتر از

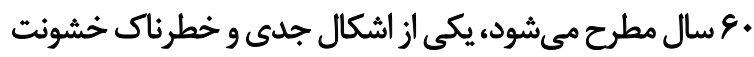


جدول ب. توزيع فراوانى ياسخ به كويههاى مقياس سوءرفتار در سالمندان مورد بررسى

\begin{tabular}{|c|c|c|c|}
\hline \multicolumn{2}{|c|}{ تعداد (دوصد) } & \multirow{2}{*}{ 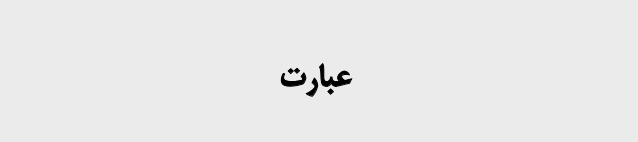 } & \multirow{2}{*}{ زيرمقياس } \\
\hline j & 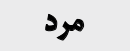 & & \\
\hline$\left.n^{e}(Y N)^{*}\right)$ & $r q(\pi / \Delta)$ & بى اعتنايعى به سالمند (حرف نزدن و ياسخ ندادن به سؤالات) & $\xi_{k}$ \\
\hline$\Lambda \cdot(r \cdot M)$ & $M(T \cdot / \mu)$ & عدم تماس حضورى يا تلفنى با سالمند & हh: \\
\hline$\mu *(11 / \Delta)$ & $\Lambda(\Delta / T)$ & كمك نكردن در حركت كردن و جابهجايى & \\
\hline $\operatorname{li}(\Delta / \varphi)$ & $P(Y / 8)$ & كمك نكردن براي مراجعه به ميزشى & $\underline{q_{0:}}$ \\
\hline $10(\Delta / V)$ & $f(T / 8)$ & كمك نكردن در تهيه و يا در مصرف دارو & $\xi_{n}^{z}$ \\
\hline$M(I T / T)$ & $9(\Delta / q)$ & عدم انجام فعاليتهاي منزل مانند نظافت و يا امور تعميراتيى & \\
\hline If $\left(\Delta / f^{e}\right)$ & $g(\% / 9)$ & عدم تأمين يول مورد نياز براي تأمين احتياجات اوليه زندكى & $\xi$ \\
\hline$M(\Lambda)$ & $r\left(V /{ }^{\prime}\right)$ & يرداخت يول توأم با منت و بى احترامى & हh: \\
\hline$r \cdot(V / M)$ & $\varepsilon(\% / q)$ & ممانعت از رفتوآمد سالمند با دوستان وخويشاوندان & \\
\hline$r \Delta(48)$ & $1 \cdot(8 / \Delta)$ & جلوكيرى از أكاه شدن سالمند از اخبار مهمه مريوط به خود & \\
\hline$M(N+)$ & $V(\varphi / 9)$ & اجازه نداشتن براي استفاده از اموال و دارايى بر اساس ميل خودش & \\
\hline $10(\Delta / N)$ & $r\left(N / M^{\prime}\right)$ & ايجاد ترس و وحشت با شكستن يا تخريب ووسايل منزل & \\
\hline TA (Q/4) & $1+(\varepsilon / \Delta)$ & فاش كردن اسرار سالمند & \\
\hline rq $(11 / 1)$ & $M(M / V)$ & عدم اهميث به شخصيث، دانش، توانايع و تجربه سالمندان & F: \\
\hline «(IF/9) & $\operatorname{Mr}($ IF/F) & سرزنش سالمثلد بلون دليل & c. \\
\hline $19(V / H)$ & $1 \cdot(\varepsilon / \Delta)$ & خطاب كردن سالمند با اسامى ناشايست، لحن نامناسب و يا بيىادباته & $E^{6}$ \\
\hline $\operatorname{st}(1 \& / 0)$ & $M(M / V)$ & داد و فرياد كشيلن بر سر سالمند & \\
\hline $10(\Delta / V)$ & $9(\Delta / 9)$ & انجام حركات توهين آميز مانند ادا يا شكلك درآوردن & \\
\hline$m(\| f / Y)$ & $\operatorname{rg}(\pi / \Delta)$ & علم بازيرداخت يول قرض كرفتهشده از سالمنلان & $a$ \\
\hline $\operatorname{IV}(g / \Delta)$ & $\mathbb{I r}(N \Delta)$ & تحميل هزينههاى زندكى بر دوش افراد مسن بلون رضايت آنها & 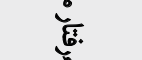 \\
\hline rq $(11 / 1)$ & $8(\% / q)$ & خوددارى از يرداخت سهميالارث سالمند & \\
\hline
\end{tabular}

جدول F. توزيع فراواني وضعيت درى سالمندان از سالمندآزارى

\begin{tabular}{|c|c|c|}
\hline ت تعداد (درصد) & ياسخ & 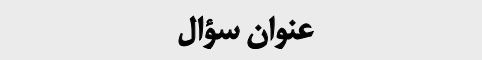 \\
\hline $\begin{array}{l}e q(Y M / V) \\
\text { IFq (FN/Y) }\end{array}$ & خيل & طرز ثلقى سالمند از بلرفتارى به عنوان سالمندآزارى \\
\hline $\begin{array}{l}|r|(\varepsilon \cdot / 1) \\
\text { AV (rq/q) }\end{array}$ & خلي & در جريان كذاثشن سوءرفثار با ديكران توسط سالمند \\
\hline $\begin{array}{c}r+\cdot(91 / V) \\
\mathbb{M}(N / r)\end{array}$ & خلى & رضايت سالمند از اعضاى خانواده \\
\hline
\end{tabular}


افزايش مشغلههاى شغلى و خانودكى فرزندان، به خصوص در در

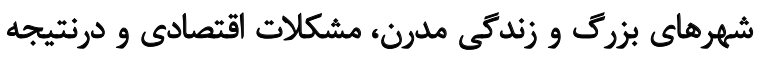

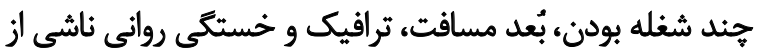

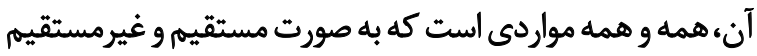

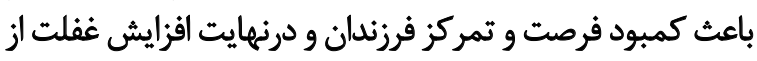
سالمندان مىشود.

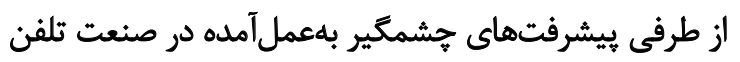

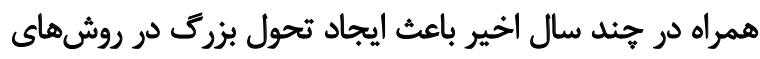

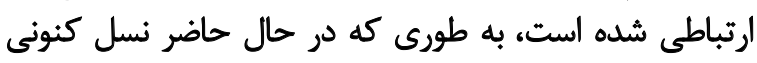

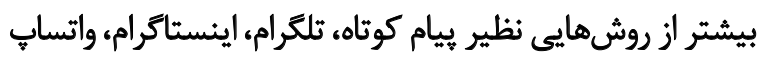

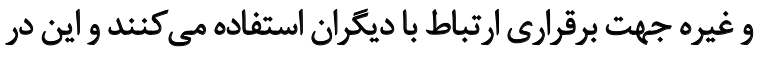

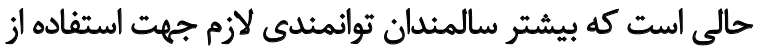

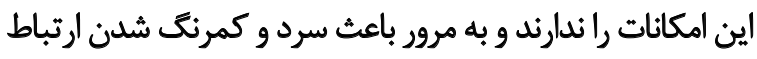

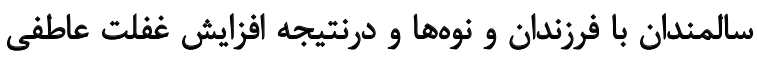

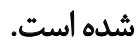

درنهايت مىتوان اين كونه نتيجه كرفت كه غفلت در بر بيشتر

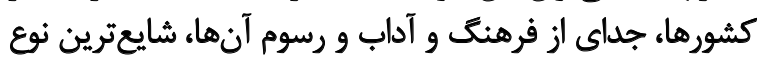

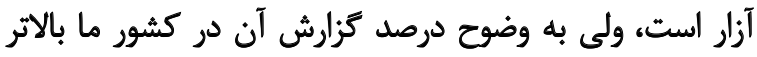

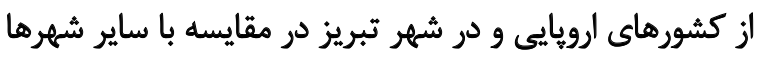

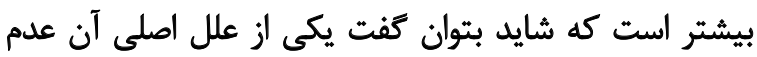

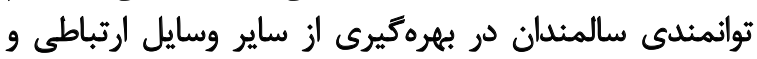

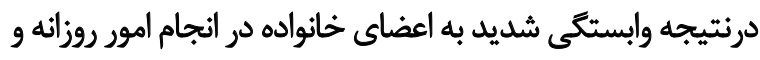
رفع نيازهاى زندكى است.

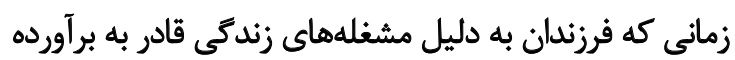

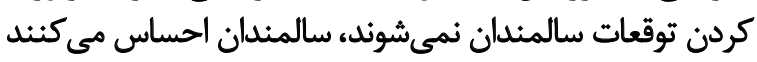

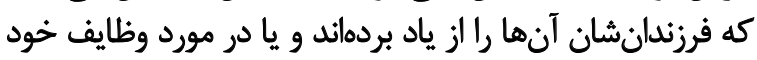

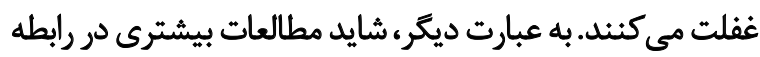

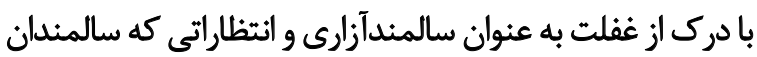

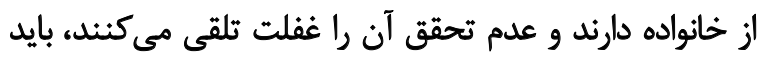

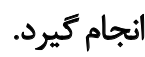

در اين مطالعه، به جز خردهمقياس سوءرفتار مالى، در ساير

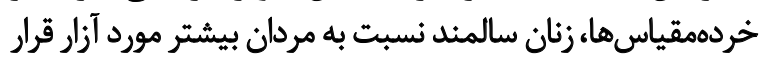

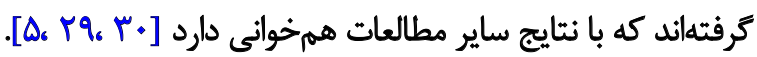

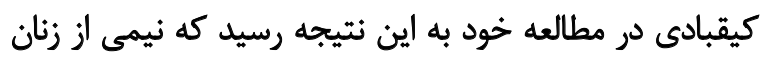

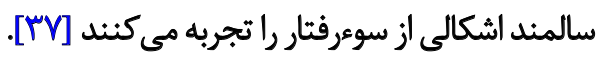
كاميلا ميلو در يورتو آلكر نشان داد بيشترين سوءاستفاده از دران

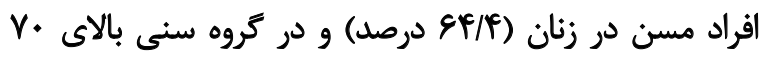

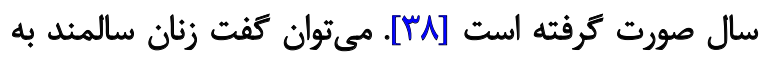

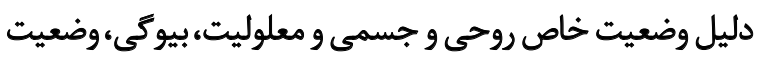

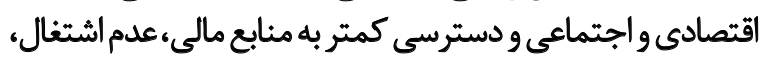

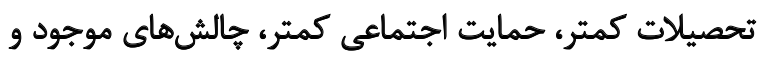

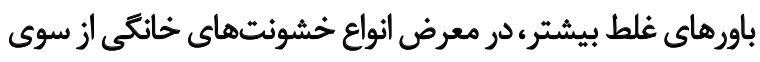

مطالعات مشابه انجامزمرفته در كشورهاي ارويايي بالاتر است.

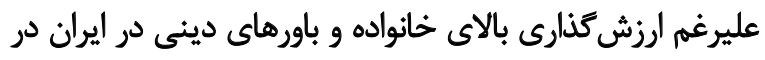

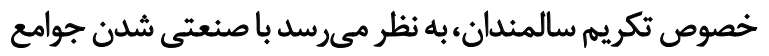

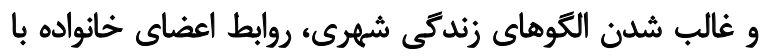

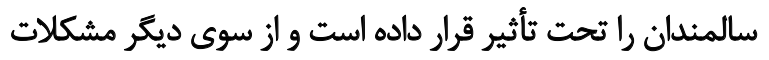

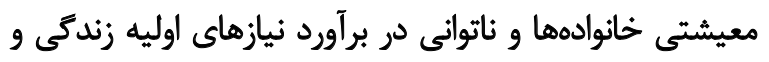

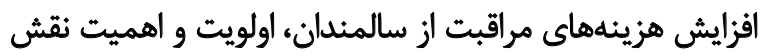

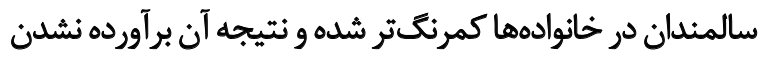
توقع بزركان و سالمندان در خانواده است.

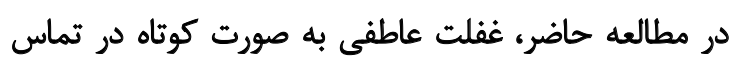

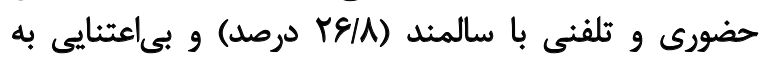

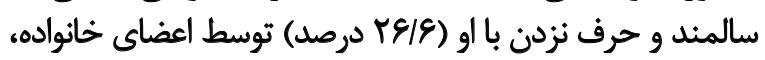

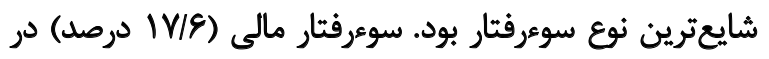

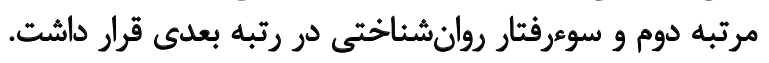

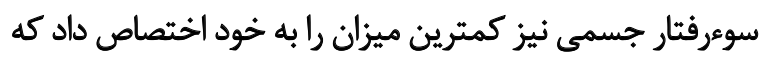

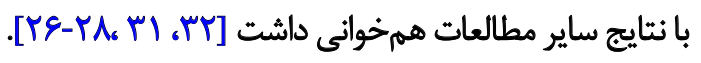

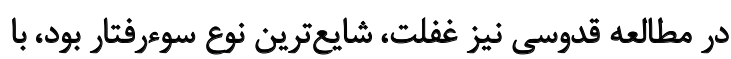

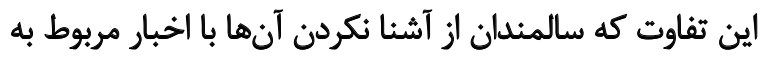

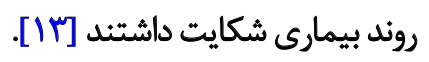

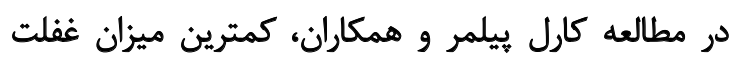

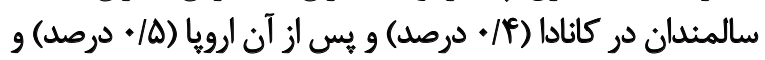

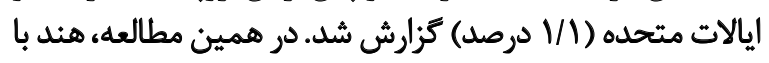

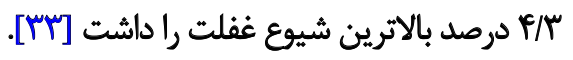

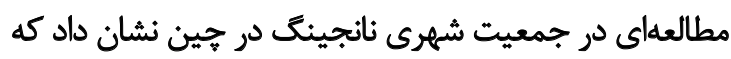

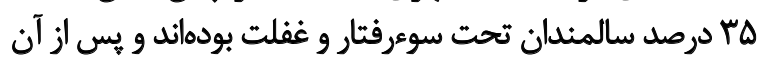

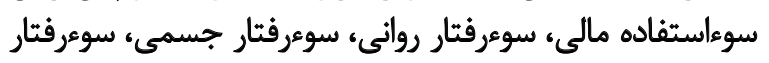

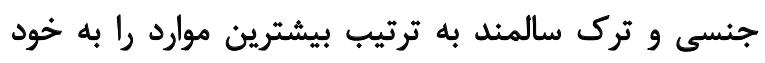

اختصاص دادهاند [TF].

در مطالعه كريستوفر و همكاران، يس از غفلت، سوءاستفاده

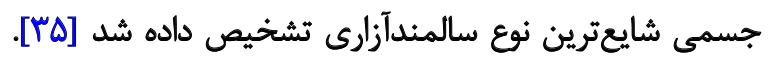

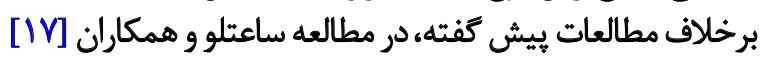

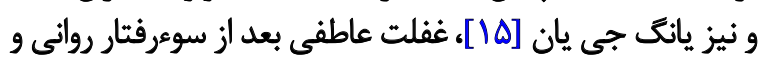

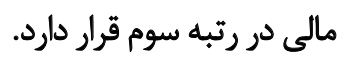

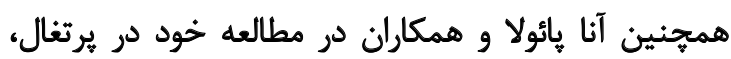

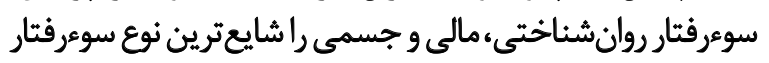

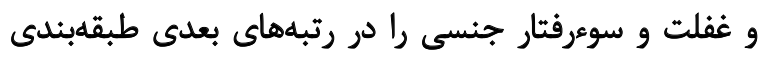

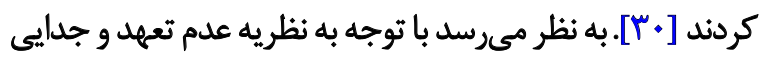

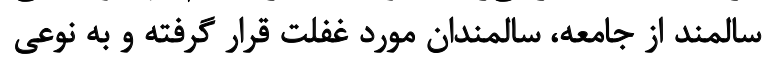

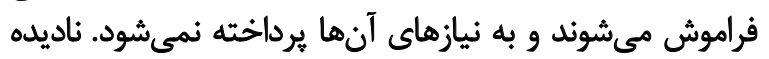

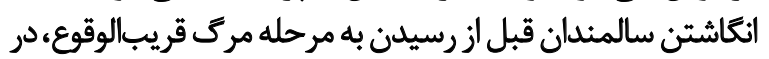

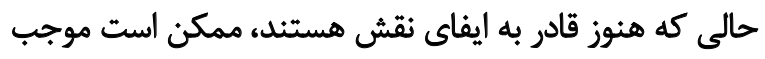

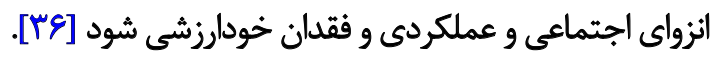


قضاوت در موارد سوءرفتار با سالمندان تعيين نشده است.

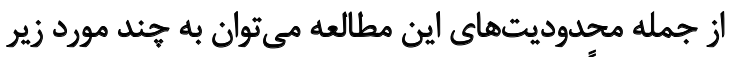

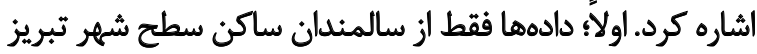

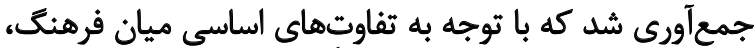

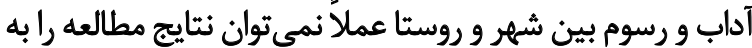
كل شهرستان تبريز تعميم داد.

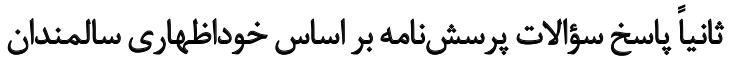

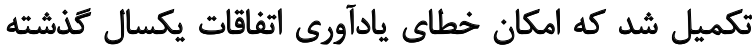

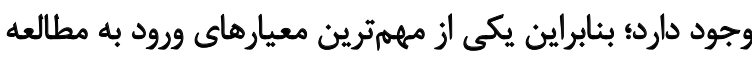
عدم وجود اختلالات شناختى در نظر كرفتئه شد.

همجنين برخى محدوديتهاى اخلاقى و فرهنتى در مورد

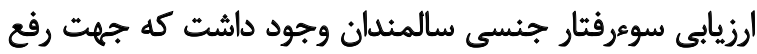

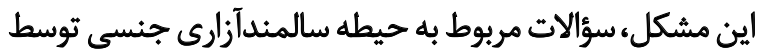

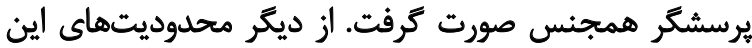

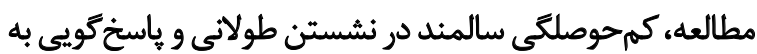

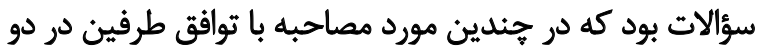

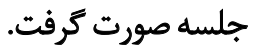

\section{تتيجلَّيرىنهايى}

مطالعه حاضر نشان مىدهد با وجود تأكيد بر احترام به

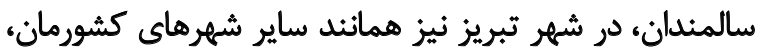

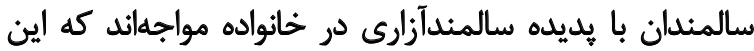

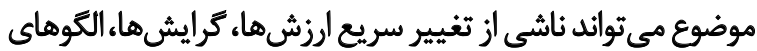

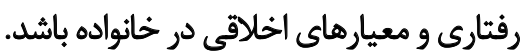

غفلت عاطفى شايعترين نوع غفلت تزارش شده و همين امر

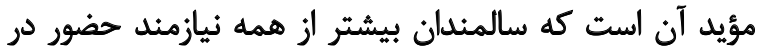

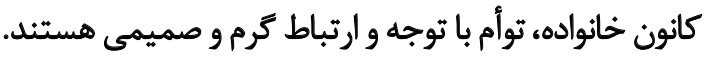

همجينين زنان نسبت به مردان در همه حيطههاى مطالعه

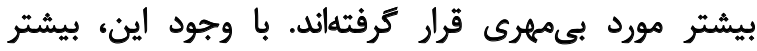

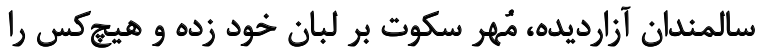

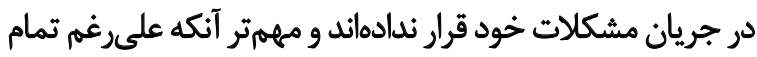

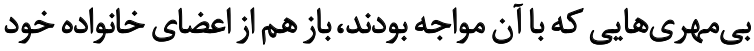

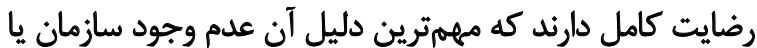

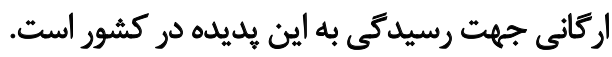

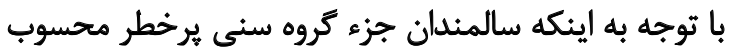

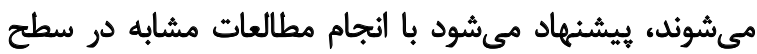

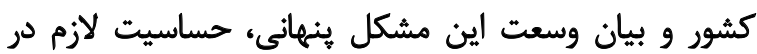

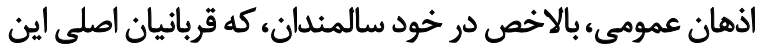

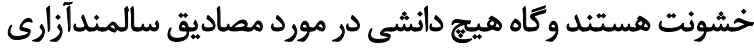

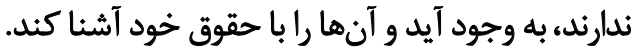

همجنين تمهيدات لازم جهت آكاهسازى خانوادهها نسبت
خانواده و اطرافيان قرار دارند و آسيبذيذيرتر هستند.

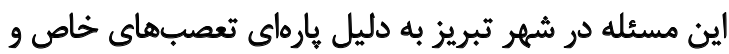

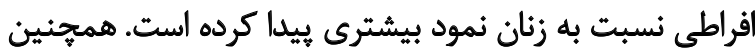

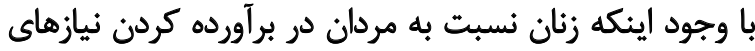

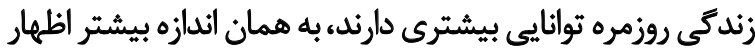

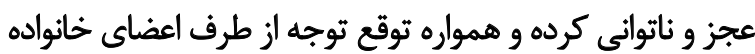

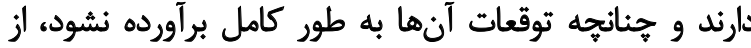
مراقبين و اعضاى خانواده اظهار نارضايتى مي كثنئد.

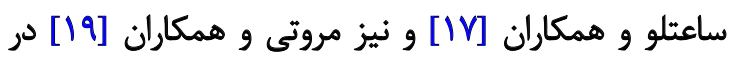

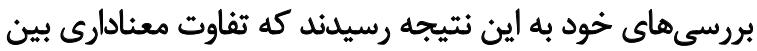

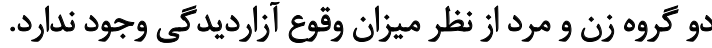

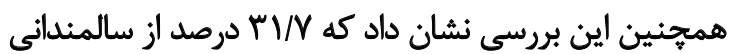

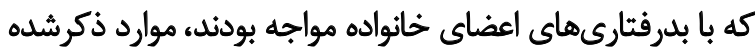

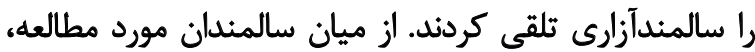

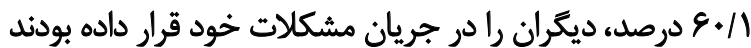

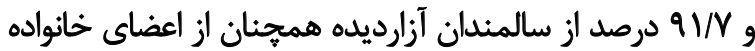
خود رضايت داشتند.

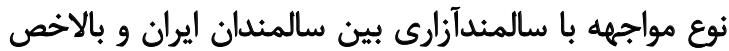

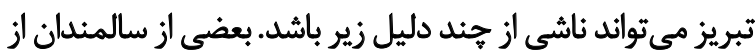

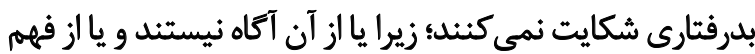

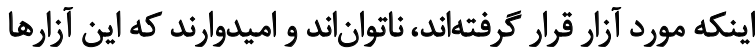

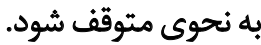

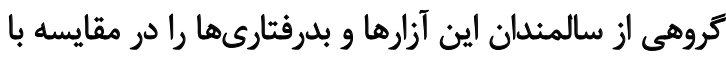

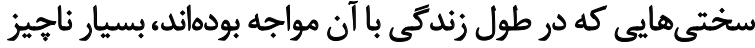

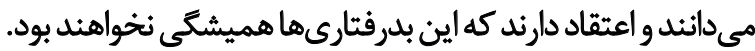

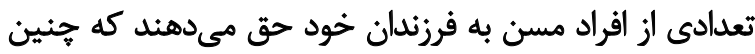

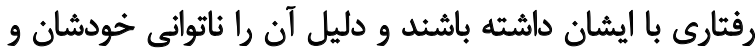

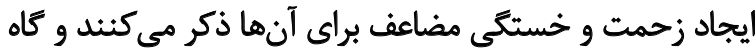

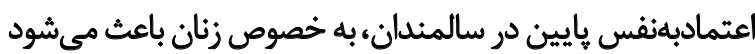
كه هيج اعتراضى به وضع موجود نداشته باشند.

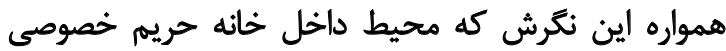

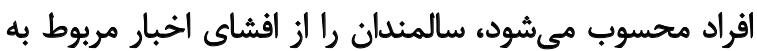

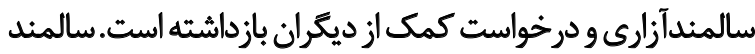

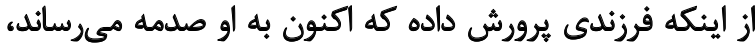

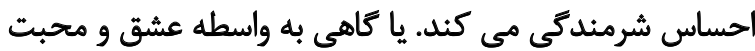

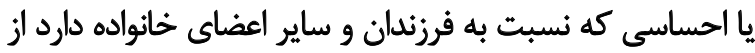

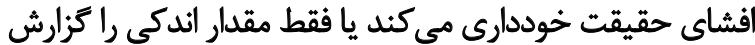

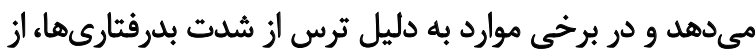

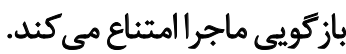

يكى از مهمترين دلايل ينهان ماندن اين مشكل در خانوادهها

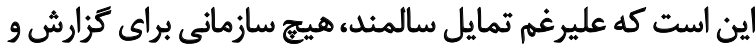




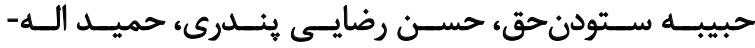

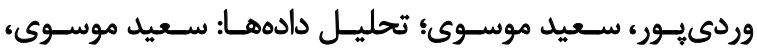

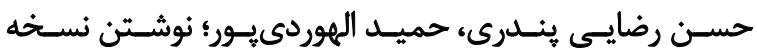

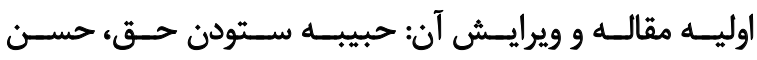

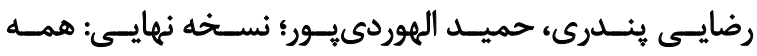

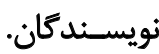

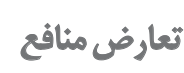

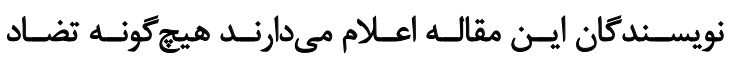

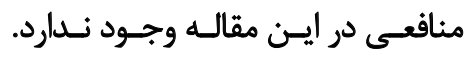

$$
\text { تشيكر و قدرواني }
$$

تمامى نويسندكان از مؤسسه راهبردهاى بازنشستخى صبا

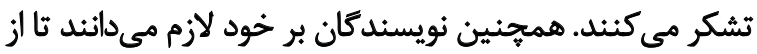

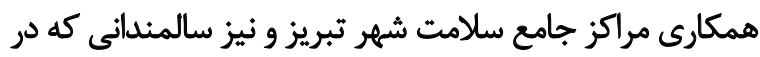

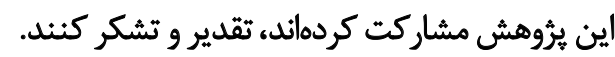

به نيازهاي جسمي، رواني، عاطفي و اجتماعى سالمندان، تغيير

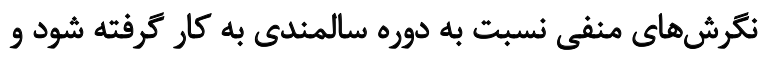

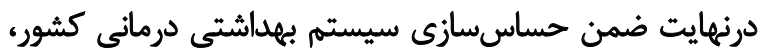

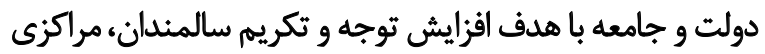

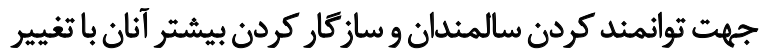
و تحولات جامعه ناشى از مدرنيته به وجود آيد.

در همين راستا افزايش تعداد كانونهاي دوستدار شهروند

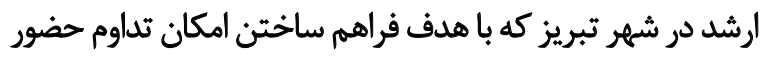

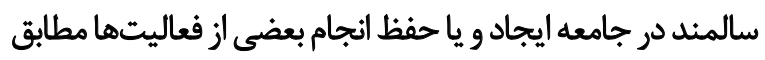

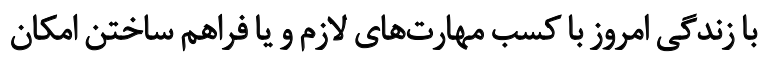

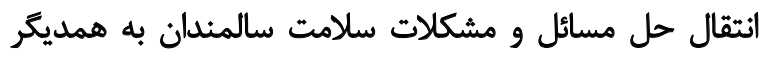

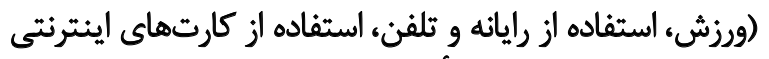

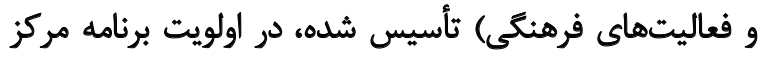
بهداشت تبريز است.

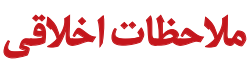

\section{بيروى از اصول اخلاق بروهش}

در اين طرح برُوهشي كه در كميته اخلاق دانشعاه علوم

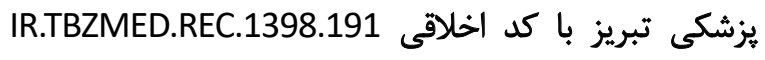
تصويب شده است به منظور رعايت ملاحظات اخلاقي، هدف أز ئزئ

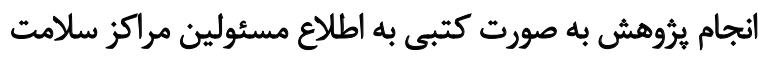

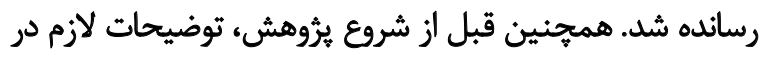

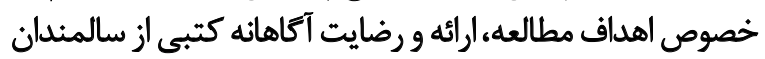
جهت شركت در مطالعه كرفته شد. مصاحبه با سالمند و تكميل

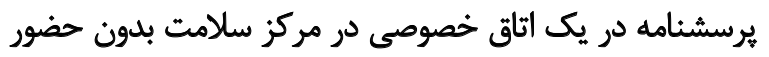

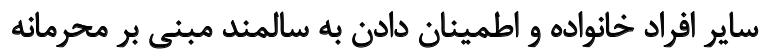

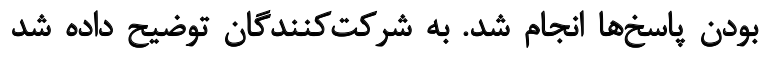

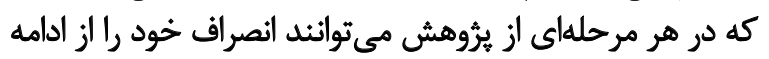

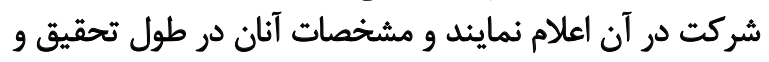

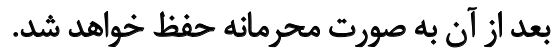

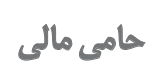

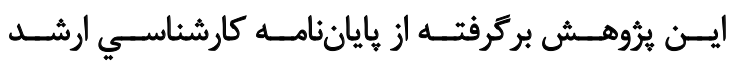

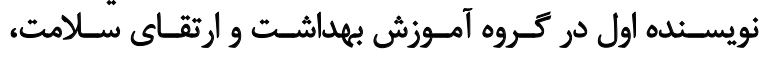

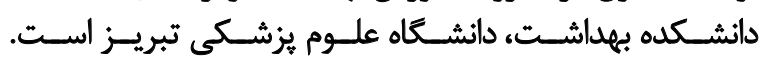

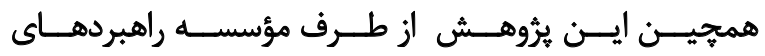

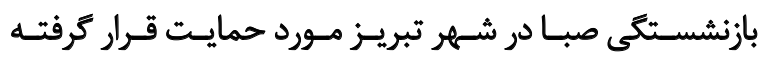

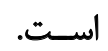

\section{مشاركت نويسندكان}

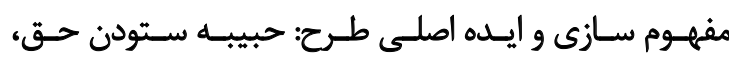

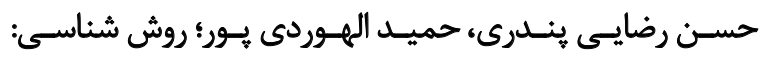




\section{References}

[1] Quinn MJ, Tomita SK. Elder abuse and neglect: Causes, diagnosis, and interventional strategies. New York: Springer Publishing Company; 1997. https://books.google.com/books/ about/Elder_Abuse_and_Neglect.html?id=NE7hb2dS5bwC

[2] World Health Organization. Elder abuse [Internet]. 2017 [Updated 2017]. Available from: https://www.who.int/ageing/ projects/elder_abuse/en/

[3] Montminy L. Older women's experiences of psychological violence in their marital relationships. Journal of Gerontological Social Work. 2005; 46(2):3-22. [DOI:10.1300/J083v46n02_02] [PMID]

[4] Morgan E, Johnson I, Sigler R. Public definitions and endorsement of the criminalization of elder abuse. Journal of Criminal Justice. 2006; 34(3):275-83. [DOI:10.1016/j.jcrimjus.2006.03.004]

[5] Heravi KM, Anousheh M, Foroughan M, Sheykhi MT, Hajizadeh E, Seyed BM, et al. [Elder abuse from the perspectives of elderly people: A phenomenological study (Persian)]. Faculty of Nursing of Midwifery Quarterly. 2008; 18(61):28-39. https://journals.sbmu.ac.ir/en-jnm/article/view/634

[6] Comijs HC, Penninx BW, Knipscheer KP, van Tilburg W. Psychological distress in victims of elder mistreatment: The effects of social support and coping. The Journals of Gerontology Series B: Psychological Sciences and Social Sciences. 1999; 54(4):P240-5. [DOI:10.1093/geronb/54B.4.P240] [PMID]

[7] Dong X, Simon M, De Leon CM, Fulmer T, Beck T, Hebert $\mathrm{L}$, et al. Elder self-neglect and abuse and mortality risk in a community-dwelling population. The Journal of the American Medical Association. 2009; 302(5):517-26. [DOI:10.1001/ jama.2009.1109] [PMID] [PMCID]

[8] Dong X, Chen R, Chang ES, Simon M. Elder abuse and psychological well-being: A systematic review and implications for research and policy-A mini review. Gerontology. 2013; 59(2):132-42. [DOI:10.1159/000341652] [PMID]

[9] Cooper C, Selwood A, Livingston G. The prevalence of elder abuse and neglect: A systematic review. Age and Ageing. 2008; 37(2):151-60. [DOI:10.1093/ageing/afm194] [PMID]

[10] Dong XQ. Elder abuse: Systematic review and implications for practice. Journal of the American Geriatrics Society. 2015; 63(6):1214-38. [DOI:10.1111/jgs.13454] [PMID]

[11] Hamberger LK, Saunders DG, Hovey M. Prevalence of domestic violence in community practice and rate of physician inquiry. Family Medicine. 1992; 24(4):283-7. [PMID]

[12] Watts C, Zimmerman C. Violence against women: Global scope and magnitude. The Lancet. 2002; 359(9313):1232-7. [DOI:10.1016/S0140-6736(02)08221-1]

[13] Ghodoosi A, Fallah Yakhdani E, Abedi HA. [Studying the instances of elder abuse and their relationship with age and sex in the hospitalized elderly (Persian)]. Iranian Journal of Forensic Medicine. 2014; 20(4\&1):367-76. http://sjfm.ir/article1-561-en.html

[14] Ho CS, Wong SY, Chiu MM, Ho R. Global prevalence of elder abuse: A metaanalysis and meta-regression. East Asian Archives of Psychiatry. 2017; 27(2):43-55. https://search.informit.org/doi/abs/10.3316/INFORMIT.903958411380250
[15] Yon Y, Mikton CR, Gassoumis ZD, Wilber KH. Elder abuse prevalence in community settings: A systematic review and meta-analysis. The Lancet Global Health. 2017; 5(2):e147-56. [DOI:10.1016/S2214-109X(17)30006-2]

[16] Denton M, Prus S, Walters V. Gender differences in health a Canadian study of the psychosocial, structural and behavioural determinants of health. Social Science \& Medicine. 2004 58(12):2585-600. [DOI:10.1016/j.socscimed.2003.09.008] [PMID]

[17] Esmat Saatlou M, Hossaini F, Sakeni Z. [Assessment of elder abuse in adult day care centers (Persian)]. Journal of Geriatric Nursing. 2015; 2(1):91-103. http://jgn.medilam.ac.ir/article1-155-en.html

[18] Molaei M, Etemad K, Taheri Tanjani P. [Prevalence of elder abuse in Iran: A systematic review and meta analysis (Persian)]. Iranian Journal of Ageing. 2017; 12(2):242-53. [DOI:10.21859/sija1202242]

[19] Morowatisharifabad MA, Rezaeipandari H, Dehghani A, Zeinali A. Domestic elder abuse in Yazd, Iran: A cross-sectional study. Health Promotion Perspectives. 2016; 6(2):104-10. [DOI:10.15171/ hpp.2016.18] [PMID] [PMCID]

[20] Walsh CA, Ploeg J, Lohfeld L, Horne J, MacMillan H, Lai D, Violence across the lifespan: Interconnections among forms of abuse as described by marginalized Canadian elders and their care-givers. British Journal of Social Work. 2007; 37(3):491-514. [DOI:10.1093/bjsw/bcm022]

[21] DeLiema M, Gassoumis ZD, Homeier DC, Wilber KH. Determining prevalence and correlates of elder abuse using promotores: Low-income immigrant Latinos report high rates of abuse and neglect. Journal of the American Geriatrics Society. 2012 60(7):1333-9. [DOI:10.1111/j.1532-5415.2012.04025.x] [PMID] [PMCID]

[22] Dong $X$, Simon MA. Urban and rural variations in the characteristics associated with elder mistreatment in a communitydwelling Chinese population. Journal of Elder Abuse \& Neglect. 2013; 25(2):97-125. [DOI:10.1080/08946566.2013.751811] [PMID] [PMCID]

[23] Perel-Levin S, World Health Organization. Discussing screening for elder abuse at primary health care level by Silvia PerelLevin. 2008. https://apps.who.int/iris/handle/10665/43523

[24] Heravi-Karimooi M, Anoosheh M, Foroughan M, Sheykhi MT, Hajizadeh E. [Designing and determining psychometric properties of the Domestic Elder Abuse Questionnaire (Persian)]. Iranian Journal of Ageing. 2010; 5(15):7-21. http://salmandj.uswr.ac.ir/ article-1-290-en.html

[25] Ribot VC, Rousseaux E, García TC, Arteaga E, Ramos ME, Alfonso M. Psychological the most common elder abuse in a Havana neighborhood. International Journal of Cuban Health and Medicine MEDICC Review. 2015; 17(2):39-43. [DOI:10.37757/ MR2015.V17.N2.9]

[26] Mohebbi L, Zahednejad S, Javadipour S, Saki A. [Domestic elder abuse in rural area of Dezful, Iran and its relation with their quality of life (Persian)]. Iranian Journal of Aging. 2016; 10(4):50-9. http://salmandj.uswr.ac.ir/article-1-888-en.html

[27] Karimi M, Elahi N. [Elderly abuse in Ahwaz city and its relationship with individual and social characteristics (Persian)] Iranian Journal of Ageing. 2008; 3(1):42-7. http://salmandj.uswr. ac.ir/article-1-84-en.html 
[28] Nori A, Rajabi A, Esmailzadeh F. [Prevalence of elder misbehavior in northern Iran (2012) (Persian)]. Journal of Gorgan University of Medical Sciences. 2015; 16(4):93-8. http:/ / goums.ac.ir/ journal/article-1-2195-en.html

[29] Ruelas-González MG, Duarte-Gómez MB, Flores-Hernández S, Ortega-Altamirano DV, Cortés-Gil JD, Taboada A, et al. Prevalence and factors associated with violence and abuse of older adults in Mexico's 2012 National Health and Nutrition Survey. International Journal for Equity in Health. 2016; 15:35. [DOI:10.1186/s12939-016-0315-y] [PMID] [PMCID]

[30] Gil AP, Kislaya I, Santos AJ, Nunes B, Nicolau R, Fernandes AA. Elder abuse in Portugal: Findings from the first national prevalence study. Journal of Elder Abuse \& Neglect. 2015; 27(3):174-95. [DOI:10.1080/08946566.2014.953659] [PMID]

[31] Rezaeipandari H, Morowatisharifabad MA, Bahrevar V, Rahaei Z, Hakimzadeh A. Relationship between abuse experience and general health among older adults in Yazd city-Iran. Elderly Health Journal. 2016; 2(1):21-6. http://ehj.ssu.ac.ir/article1-51-en.html

[32] Yunus RM, Wazid SW, Hairi NN, Choo WY, Hairi FM, Sooryanarayana R, et al. Association between elder abuse and poor sleep: A cross-sectional study among rural older Malaysians. PLoS One. 2017; 12(7):e0180222. [DOI:10.1371/journal. pone.0180222] [PMID] [PMCID]

[33] Pillemer K, Burnes D, Riffin C, Lachs MS. Elder abuse: Global situation, risk factors, and prevention strategies. The Gerontologist. 2016; 56(Suppl 2):S194-205. [DOI:10.1093/geront/gnw004] [PMID] [PMCID]

[34] Dong X, Simon MA, Gorbien M. Elder abuse and neglect in an urban Chinese population. Journal of Elder Abuse \& Neglect. 2007; 19(3-4):79-96. [DOI:10.1300/J084v19n03_05] [PMID]

[35] Evans CS, Hunold KM, Rosen T, Platts-Mills TF. Diagnosis of elder abuse in US emergency departments. Journal of the American Geriatrics Society. 2017; 65(1):91-7. [DOI:10.1111/jgs.14480] [PMID] [PMCID]

[36] Cumming E, Dean LR, Newell DS, McCaffrey I. Disengagement-a tentative theory of aging. Sociometry. $1960 ; 23(1): 23-35$. [DOI:10.2307/2786135]

[37] Keyghobadi F, Moghaddam Hosseini V, Keyghobadi F, Rakhshani MH. [Prevalence of elder abuse against women and associated factors (Persian)]. Journal of Mazandaran University of Medical Sciences. 2014; 24(117):125-32. http://jmums.mazums. ac.ir/article-1-4447-en.html

[38] Santos CM, Marchi RJ, Martins AB, Hugo FN, Padilha DM, Hilgert JB. The prevalence of elder abuse in the Porto Alegre metropolitan area. Brazilian Oral Research. 2013; 27(3):197-202. [DOI:10.1590/S1806-83242013005000011] [PMID] 